\title{
The Dutch Pancreas Biobank Within the Parelsnoer Institute
}

\section{A Nationwide Biobank of Pancreatic and Periampullary Diseases}

\author{
Marin Strijker, MD, * Arja Gerritsen, $M D, P h D,{ }^{*}$ Jony van Hilst, MD, MSc, * Maarten F. Bijlsma, PhD, $†$ \\ Bert A. Bonsing, MD, PhD, + Lodewijk A. Brosens, MD, PhD, $\$ / /$ Marco J. Bruno, MD, PhD, $\mid$ \\ Ronald M. van Dam, MD, PhD,\# Frederike Dijk, PhD, ** Casper H. van Eijck, MD, PhD, $\dagger \dagger$ \\ Arantza Farina Sarasqueta, MD, PhD, $+\neq$ Paul Fockens, MD, PhD, $\$$ S Michael F. Gerhards, MD, PhD,/I/ \\ Bas Groot Koerkamp, MD, PhD, †† Erwin van der Harst, MD, PhD, 9 I Ignace H. de Hingh, MD, PhD, \#\# \\ Jeanin E. van Hooft, MD, PhD, MBA, §§ Clément J. Huysentruyt, MD, *** Geert Kazemier, MD, PhD, †† \\ Joost M. Klaase, MD, PhD, $+1+\$ \$ \$$ Cornelis J. van Laarhoven, MD, PhD, //I/I \\ Hanneke W. van Laarhoven, MD, PhD, PhD, 199 Mike S. Liem, MD, PhD, t+t
}

Vincent E. de Meijer, MD, PhD, MSc, $\oint \oint \oint$ L. Bengt van Rijssen, MD, * Hjalmar C. van Santvoort, MD, PhD,\#\#\#

Mustafa Suker, MD, †† Judith H. Verhagen, PhD, $\$ Joanne Verheij, MD, PhD, ** Hein W. Verspaget, PhD, ****

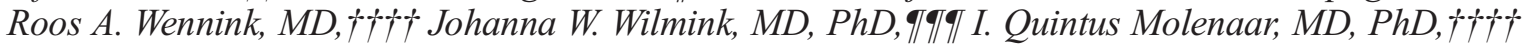

Marja A. Boermeester, MD, PhD,ttt Olivier R. Busch, MD, PhD, *

and Marc G. Besselink, MD, PhD, MSc, *

for the Dutch Pancreatitis Study Group and Dutch Pancreatic Cancer Group

\begin{abstract}
Objectives: Large biobanks with uniform collection of biomaterials and associated clinical data are essential for translational research. The Netherlands has traditionally been well organized in multicenter clinical research on pancreatic diseases, including the nationwide multidisciplinary Dutch Pancreatic Cancer Group and Dutch Pancreatitis Study Group. To enable highquality translational research on pancreatic and periampullary diseases, these groups established the Dutch Pancreas Biobank.

Methods: The Dutch Pancreas Biobank is part of the Parelsnoer Institute and involves all 8 Dutch university medical centers and 5 nonacademic hospitals. Adult patients undergoing pancreatic surgery (all indications) are eligible for inclusion. Preoperative blood samples, tumor tissue from resected specimens, pancreatic cyst fluid, and follow-up blood samples are collected. Clinical parameters are collected in conjunction with the mandatory Dutch Pancreatic Cancer Audit.
\end{abstract}

Results: Between January 2015 and May 2017, 488 patients were included in the first 5 participating centers: 4 university medical centers and 1

From the *Department of Surgery, Cancer Center Amsterdam, Academic Medical Center, Amsterdam ; † Laboratory for Experimental Oncology and Radiobiology, Center for Experimental and Molecular Medicine, Academic Medical Center and Cancer Center Amsterdam, Amsterdam; \$Department of Surgery, Leiden University Medical Center, Leiden; §Department of Pathology, University Medical Center, Utrecht; ||Department of Pathology, Radboud University Medical Center, Nijmegen; $\bullet$ Department Gastroenterology and Hepatology, Erasmus Medical Center, Rotterdam; \#Department of Surgery, Maastricht University Medical Center, Maastricht; **Department of Pathology, Cancer Center Amsterdam, Academic Medical Center, Amsterdam; ††Department of Surgery, Erasmus Medical Center, Rotterdam; +\$Department of Pathology, Leiden University Medical Center, Leiden; §§Department of Gastroenterology, Cancer Center Amsterdam, Amsterdam Institute for Gastroenterology and Metabolism, Academic Medical Center Amsterdam; \|||Department of Surgery, OLVG, Amsterdam; $\uparrow \mid$ Department of Surgery, Maasstad Hospital, Rotterdam; \#\#Department of Surgery and $* * *$ Department of Pathology, Catharina Hospital, Eindhoven; ††Department of Surgery, Cancer Center Amsterdam, VU Medical Center, Amsterdam; +H Department of Surgery, Medisch Spectrum Twente, Enschede; $\S \S \S$ Department of Surgery, University of Groningen and University Medical Center Groningen, Groningen; ||W||Department of Surgery, Radboud University Medical Center, Nijmegen; 9 qा Department of Medical Oncology, Cancer Center Amsterdam, Academic Medical Center, Amsterdam; \#\#\#Department of Surgery, St Antonius Hospital, Nieuwegein; ****Parelsnoer Institute, Utrecht; $+\dagger+$ Department of Surgery, University Medical Center, Utrecht; and $+\$+t D e-$ partment of Surgery, Academic Medical Center, Amsterdam, the Netherlands. Received for publication June 26, 2017; accepted January 18, 2018. nonacademic hospital. Over 2500 samples were collected: 1308 preoperative blood samples, 864 tissue samples, and 366 follow-up blood samples. Conclusions: Prospective collection of biomaterials and associated clinical data has started in the Dutch Pancreas Biobank. Subsequent translational research will aim to improve treatment decisions based on disease characteristics.

Key Words: pancreatic cancer, chronic pancreatitis, periampullary tumors, biobank, bioresources, personalized medicine

(Pancreas 2018;47: 495-501)

D ancreatic cancer is one of the most lethal cancers, with a 5 -year survival rate of $6 \% .{ }^{1}$ Surgical resection combined with adjuvant chemotherapy shows the best outcomes with 5 -year survival rates of $20 \%{ }^{2,3}$ However, only $15 \%$ to $20 \%$ of patients present with a (potentially) resectable cancer. ${ }^{4}$ Currently available

Address correspondence to: Marc G. Besselink, MD, PhD, MSc, Department of Surgery, Cancer Center Amsterdam, Academic Medical Center, G4-196, PO Box 22660, 1100 DD Amsterdam, the Netherlands

(e-mail: m.g.besselink@amc.nl); Marin Strijker, MD, Department of Surgery, Cancer Center Amsterdam, Academic Medical Center, G4-130, PO Box 22660, 1100 DD Amsterdam, the Netherlands (e-mail: m.strijker@amc.nl).

Conflicts of Interest and Source of Funding: The Dutch Pancreas Biobank received funding from the Dutch Cancer Society (KWF Kankerbestrijding; grant no. UVA2013-5842)

The authors declare no conflict of interest.

Collaborators: Ralph H. Hruban, MD, Department of Pathology, The Sol Goldman Pancreatic Cancer Research Center, The Johns Hopkins University School of Medicine, Baltimore, MD; Department of Oncology, The Sol Goldman Pancreatic Cancer Research Center, The Johns Hopkins University School of Medicine, Baltimore, MD.

David A. Tuveson, MD, PhD, Cold Spring Harbor Laboratory, Cold Spring Harbor, NY; Lustgarten Foundation Pancreatic Cancer Research Laboratory, Cold Spring Harbor, NY.

William Greenhalf, PhD, National Institute for Health Research (NIHR) Liverpool Pancreas Biomedical Research Unit, Royal Liverpool University Hospital, Liverpool, UK

David K. Chang, MBBS, PhD, Wolfson Wohl Cancer Research Centre, University of Glasgow, UK.

Copyright (C) 2018 Wolters Kluwer Health, Inc. All rights reserved.

DOI: 10.1097/MPA.0000000000001018 
chemotherapy strategies have only a limited effect on survival and quality of life, both in adjuvant and palliative setting., 5

In various types of cancers, the development of new genetic and molecular tools has led to a more tailored treatment of patients based on selective tumor features. ${ }^{7-9}$ Despite the increasing knowledge of tumor biology in pancreatic cancer, no translation into effective targeted treatment strategies has been achieved so far. ${ }^{10}$ Several targeted agents for pancreatic cancer have been evaluated in clinical trials, but only the HER1/EGFR tyrosine kinase inhibitor erlotinib (in combination with gemcitabine) for locally advanced or metastatic pancreatic cancer has been approved by the Food and Drug Administration. ${ }^{11}$ Improved selection of patients who may benefit from targeted therapies might lead to better outcomes. Currently, no validated biomarkers to guide personalized treatment decisions are available for erlotinib or other targeted therapies in pancreatic cancer. ${ }^{10,12}$

Pancreatic cancer shares biological characteristics with the 3 other periampullary tumors (ie, distal cholangiocarcinoma, duodenal carcinoma, and ampulla of Vater carcinoma), but important differences exist between their mutational profiles, biological behavior, and response to chemotherapy. ${ }^{13}$ Research initiatives on molecular characterization and tailored treatment of periampullary tumors are currently limited, but urgently needed given the limited survival. Moreover, differentiation between these tumor types may be difficult, even on final pathology. This, while the correct diagnosis of the tumor type is extremely important for treatment decisions, as adjuvant chemotherapy is only indicated in case of pancreatic cancer and, based on recent evidence, distal cholangiocarcinoma. ${ }^{14}$ Also in palliative setting, chemotherapy regimens differ between pancreatic cancer and other periampullary cancers. ${ }^{15,16}$

Chronic pancreatitis $(\mathrm{CP})$ is a benign but debilitating disease associated with severe chronic abdominal pain and complications such as pseudocysts and bile duct or duodenal obstruction. Treatment is focused on pain management and exo- and endocrine insufficiency. ${ }^{17}$ In severe cases, surgery is effective in relieving pain or to treat complications. ${ }^{18}$ To further improve risk management and treatment strategies, a better understanding of the molecular mechanisms and genetic factors in CP is essential. ${ }^{17,19,20}$

In conclusion, more research should focus on genetics and biomarkers if care for these pancreatic diseases is to be improved. An important problem is the lack of high-quality biomaterials. ${ }^{2}$ Biomaterials collected without a well-established biobank protocol often vary in the execution of collection, processing, and storage. ${ }^{2,21,22}$ Moreover, clinical data are often not available or not properly associated with the samples. This is less of a problem within clinical trials, but materials collected during clinical trials often are not usable for other projects because of the narrow informed consent or the selected subset of patients included in the trial. ${ }^{2,22}$ For these reasons, large-scale biobanks with a broad informed consent, which are linked to clinical data, and in which samples are uniformly collected, processed, and stored, are essential for future molecular and genetic research in pancreatic diseases. ${ }^{2,21}$

In the Netherlands, a strong nationwide platform for multicenter multidisciplinary research on pancreatic diseases has been established over a period of decades within the pancreatic cancer and pancreatitis research groups [Dutch Pancreatic Cancer Group (DPCG) and Dutch Pancreatitis Study Group (DPSG)]. Moreover, a nationwide collaboration for the establishment, expansion, and optimization of clinical biobanks for scientific research is provided by the Parelsnoer Institute (PSI). To facilitate future translational research on pancreatic diseases, the DPCG and DPSG established the Dutch Pancreas Biobank within the Parelsnoer collaboration.

The aim of the current study was not only to inform other researchers about the research opportunities of our biobank but also to provide a framework to those interested in biobanking or launching a similar initiative.

\section{MATERIALS AND METHODS}

\section{Biobank Design}

The Dutch Pancreas Biobank is incorporated in the PSI (www.parelsnoer.org). ${ }^{23}$ The PSI is a nationwide network founded by the Dutch Federation of University Medical Centers (NFU) in 2007. During the past decade, biobanks have been established for several diseases (eg, gastroesophageal cancer, kidney failure, and cerebral stroke). ${ }^{24-26}$ The PSI framework is designed to consider all relevant legal and ethical standards, including privacy and informed consent procedures. All PSI biobanks share the same central infrastructure (including IT system), striving for optimal harmonization of data collection. The PSI standard operating procedures are used by all participating centers to ensure high-quality biomaterials and harmonized collecting, processing, and storing processes. ${ }^{27}$

The Dutch Pancreas Biobank is part of the Dutch Pancreatic Cancer Project (PACAP), coordinated by the DPCG, which aims to improve outcomes of patients with pancreatic cancer. The PACAP includes a mandatory nationwide prospective registration of clinical data and patient-reported outcomes and the collection of biomaterials.

\section{Funding}

The Dutch government, NFU, and all 8 university medical centers financed the formation of PSI. Because all biobanking facilities have been established, costs for collection and storage of all PSI biobanks are covered by the individual participating hospitals. Moreover, PACAP receives funding from the Dutch Cancer Society (KWF Kankerbestrijding; grant no. UVA 2013-5842).

\section{Eligibility and Informed Consent}

All patients with an indication for pancreatic surgery are eligible for inclusion. This includes both patients with $\mathrm{CP}$ and patients with pancreatic or periampullary tumors [pancreatic cancer, distal cholangiocarcinoma, duodenal carcinoma, ampullary carcinoma, neuroendocrine tumors, metastases of other tumors to the pancreas, and cystic lesions (intraductal papillary mucinous neoplasm, mucinous cystic neoplasms, and serous cystic neoplasms)].

The biobank protocol and regulations were approved by the PSI, the Biobank Ethics Committee of the Academic Medical Center, Amsterdam, and by the institutional review boards (IRBs) of all the participating centers. Only patients able and willing to provide informed consent are included. Informed consent covers collection and use of encoded clinical data; collection, storage, and use of biomaterials; permission to obtain data from the municipal register, the general practitioner, and Statistics Netherlands (CBS); and permission to be approached in the future to provide extra data or biomaterials.

\section{Data Collection}

Clinical data of patients with pancreatic and periampullary disease are collected in conjunction with the Dutch Pancreatic Cancer Audit for patients with pancreatic and periampullary tumors and the Dutch Chronic Pancreatitis Registry (CARE) for patients with CP.

Clinical data registered in the Dutch Pancreas Biobank include medical history, intoxications, family history, physical examination, diagnostics, surgical procedures, pathology, and follow-up. For patients with $\mathrm{CP}$, additional specific items on pain, complications, and interventions are collected. 
Clinical data and biomaterial information are stored in Project Manager Internet Server (ProMISEe), the central database of PSI (Fig. 1). Data are pseudonymized via a Trusted Third Party. Biomaterial data include collection date, type of material, standard operating procedure deviations, DNA quality, and availability of the samples.

\section{Collection of Biomaterials}

Before surgery, venous blood samples are collected: a 10-mL serum clot tube and one or two 10-mL EDTA plasma tubes (Table 1). One of the EDTA plasma tubes and the serum tube are centrifuged, and serum and plasma are stored in $0.5-\mathrm{mL}$ aliquots at $-80^{\circ} \mathrm{C}$. The other 10-mL ETDA plasma tube or the pellet from the first EDTA plasma tube is used for genomic DNA isolation from whole blood. DNA is isolated within 4 weeks and stored at $4^{\circ} \mathrm{C}$ or $-20^{\circ} \mathrm{C}$ or less. DNA is stored as stock solutions, which will be diluted for actual use. DNA concentration and the quality of the stock solution (using, respectively, OD $260 \mathrm{~nm}$ and OD ratio 260-280 or fluorimetry) are measured. In the case of a DNA concentration less than $50 \mu \mathrm{g} / \mathrm{mL}$, blood for DNA isolation will be collected again during follow-up.

In the Netherlands, pancreatic resections are currently performed in 18 centers, of which 13 centers currently participate in the Dutch Pancreas Biobank. In case of pancreatic or periampullary tumors, the resected specimen is immediately transported to the pathology department in an unfixed state. First, the pathologist takes the routine samples from the specimen required for diagnosis. Subsequently, samples $\left(0.5 \mathrm{~cm}^{3}\right)$ for storage in the biobank are collected, without interfering with standard tissue processing for diagnostic purposes. Two samples of the tumor, one of the normal pancreatic tissue and one of the duodenum (in the case of pancreatoduodenectomy) or spleen (in the case of distal pancreatectomy with splenectomy), are collected. In addition, if possible, a macroscopically involved lymph node or metastasis is sampled. In the case of a pancreatic cyst, cyst fluid is collected. For patients with $\mathrm{CP}$, samples $\left(0.5 \mathrm{~cm}^{3}\right)$ from the pancreatic head, body, and tail are collected during surgery. All samples are snap frozen, usually within 1.5 hours after resection, and the samples are stored at $-80^{\circ} \mathrm{C}$. Follow-up is carried out according to the local standard of the participating hospitals. Biomaterials are collected at several time points during follow-up (Table 1).

\section{Research Proposals and Use of Samples}

To ensure that the collected biomaterials are used for highquality research projects, a workflow for the review process has been established. Research proposals are submitted to the coordinator of the Dutch Pancreas Biobank and subsequently evaluated by a dedicated scientific committee. When approved by the scientific committee, the Dutch Pancreas Biobank scientific board has to approve the proposal as well. This scientific board consists of one member from all specialties involved in the treatment of patients with pancreatic diseases (surgery, medical oncology, gastroenterology, pathology, radiology, radiotherapy, and translational research) from each participating center. Hereafter, approval of the IRB from the centers of which samples are used will be acquired. Subsequently, the samples will be provided to the researcher within 4 weeks from the official request to the biobank.

\section{RESULTS}

Currently, 13 centers collaborate in the Dutch Pancreas Biobank. Four university medical centers and 1 nonacademic hospital have started with the collection of biomaterials. In 5 more centers, IRB approval has been obtained. Between February 2015 and May 2017, 488 patients have been included from the 5 centers, with over
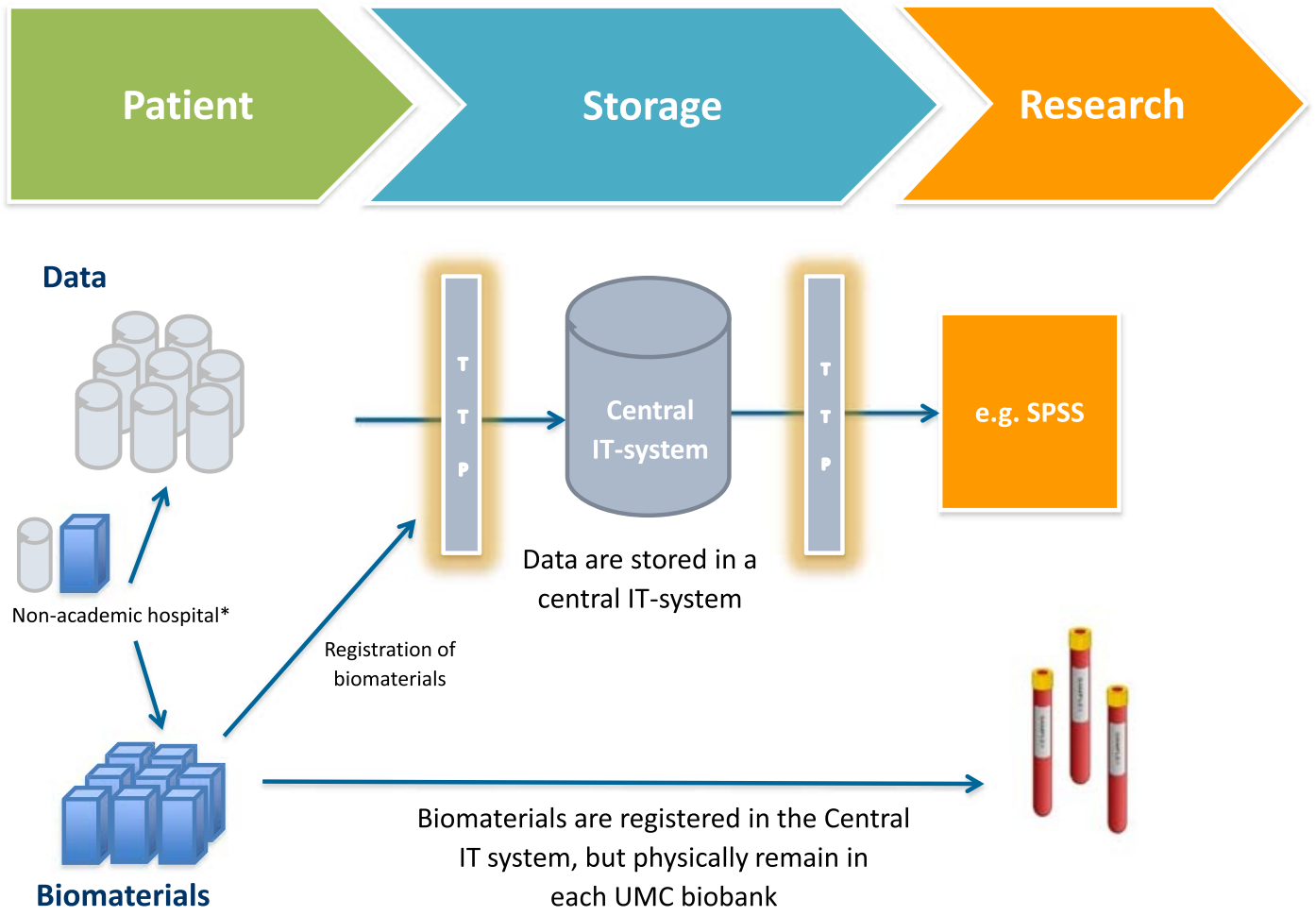

FIGURE 1. Workflow of data and biomaterial collection in the Dutch Pancreas Biobank. *Data and biomaterials collected in nonacademic hospitals are coded and transferred to one of the 8 UMCs. Editor's note: A color image accompanies the online version of this article. 
TABLE 1. Overview of Collection Protocol of the Dutch Pancreas Biobank

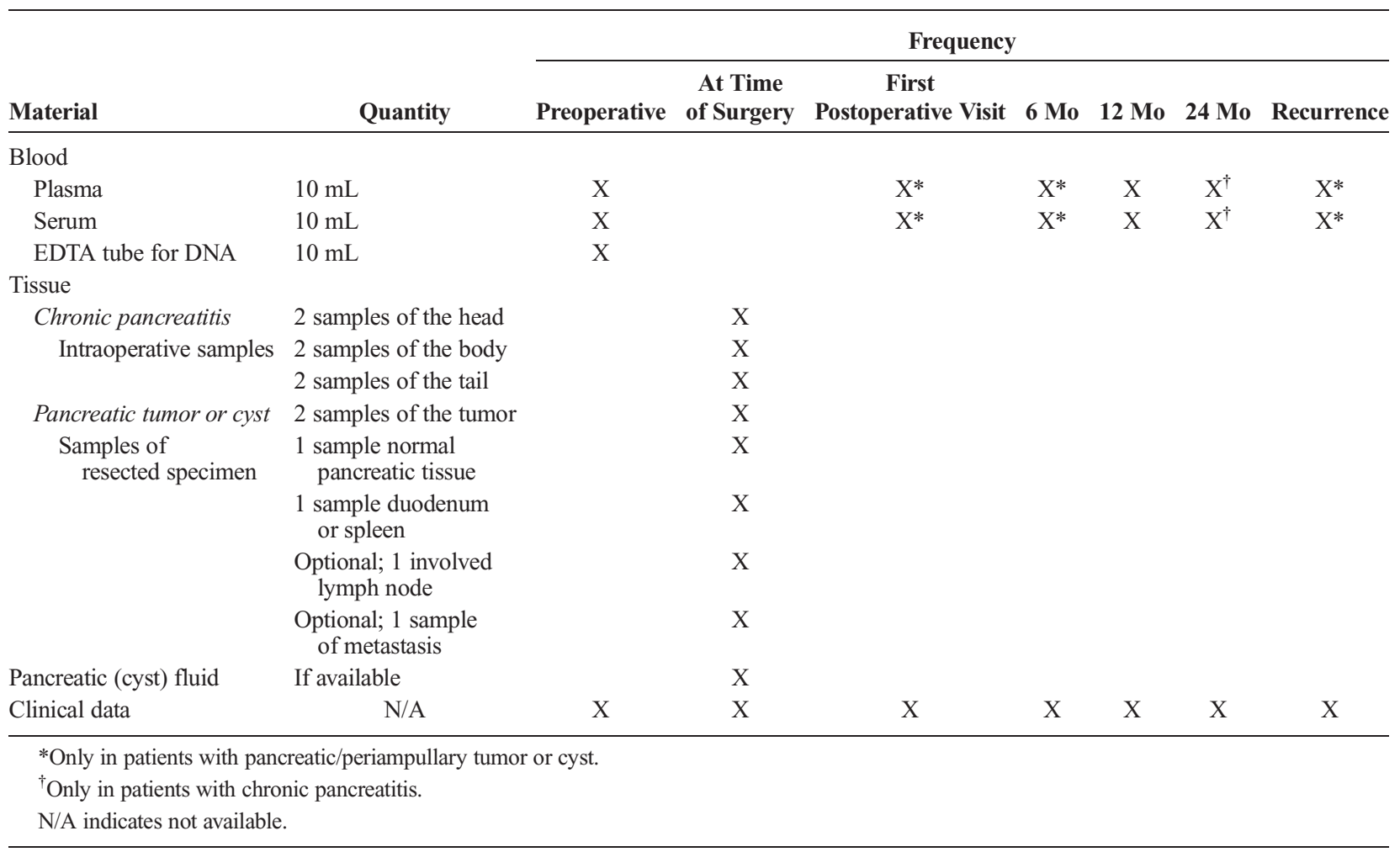

2500 samples collected: 1308 preoperative blood samples, 864 tumor tissue samples, 11 cyst fluid samples, and 366 follow-up blood samples.

\section{Baseline Characteristics}

Baseline characteristic of the patients who underwent resection in 2015 and 2016 are shown in Table 2. The majority of the included patients underwent a resection with curative intent because of suspected (pre) malignant disease $(n=275,82.8 \%)$. The most frequent histopathological diagnosis in this group were pancreatic cancer $(n=119,43.3 \%)$, pancreatic neuroendocrine tumor $(\mathrm{NET})(\mathrm{n}=27,9.8 \%)$, distal cholangiocarcinoma $(\mathrm{n}=26$, $9.5 \%)$, and carcinoma of the ampulla of Vater $(n=25,9.1 \%)$. At the time of preoperative blood collection and during tumor tissue collection, $90.6 \%(n=301)$ of the patients were systemic treatment naive. Adjuvant chemotherapy was discussed in the majority $(\mathrm{n}=97,81.5 \%)$ of patients with pancreatic cancer. In patients undergoing a pancreatic resection because of $\mathrm{CP}$, the etiology was alcoholic in $57.7 \%(\mathrm{n}=15)$ of the patients. The indication for surgery was pain in $84.6 \%(n=22)$ of patients, and the most frequently performed procedure was a Frey procedure $(n=11,42.3 \%)$.

\section{DISCUSSION}

A nationwide biobank has been established in the Netherlands that aims to facilitate translational research toward more personalized medicine for patients with pancreatic disease. More than 2500 samples from 488 patients have been collected between February 2015 and May 2017, and the biobank is rapidly expanding.

In the last decade, several research groups acknowledged the importance of large-scale multicenter biobanks. The PANcreatic Disease ReseArch (PANDoRA) consortium retrospectively included clinical data and DNA samples from 2220 patients with pancreatic cancer from 15 research groups across 6 countries. ${ }^{28}$ The consortium studied single nucleotide polymorphisms in patients with pancreatic cancer and was able to identify new pancreatic cancer susceptibility loci. ${ }^{28}$ The Australian Pancreatic Cancer Genome Initiative BioResources (APGI; Australian contribution to the International Cancer Genome Consortium) is another example of such effort $^{29}$; the APGI Bioresource supported the genomic analyses to identify molecular subtypes of pancreatic cancer and to describe the genome landscape of pancreatic NETs. ${ }^{30-33}$ The Pancreatic Cancer Research Fund Tissue Bank established in the UK is a multicenter project similar to the Dutch Pancreas Biobank. In the Pancreatic Cancer Research Fund Tissue Bank, tissue, blood, saliva, and urine are collected in 6 centers. ${ }^{22}$ Other large biobanking projects on pancreatic cancer include the biobank of the Liverpool Pancreas Biomedical Research Unit (now stored as part of Liverpool Good Clinical Practice Laboratory facility) and the Johns Hopkins Tissue Resource (Gastrointestinal Cancer Specialized Program of Research Excellence). ${ }^{34,35}$ The Dutch Pancreas Biobank seems to stand out because of its long-term follow-up along with biomaterials and its coverage of the majority of the country.

While establishing and running this large and logistically complex nationwide project, we faced several challenges. First is the logistics; the infrastructure for processing, storing, and labeling of biomaterials is provided by the PSI, but each center has to set up their own clinical workflows. This includes obtaining informed consent, collection of blood samples, transportation of pathology specimens immediately after resection, collection of tissue samples, proper storage of all biomaterial, and registration of/association with clinical parameters. This is a very time-consuming process and may be challenging to implement in daily practice. We have found that sharing experiences between centers help to establish 
TABLE 2. Baseline Characteristics of 358 Patients Included in the Dutch Pancreas Biobank in 2015-2016

\begin{tabular}{|c|c|c|}
\hline Characteristics & $\begin{array}{l}\text { (Pre-)Malignant } \\
\text { Disease }(n=332)\end{array}$ & $\begin{array}{c}C P \\
(n=26)\end{array}$ \\
\hline Age at inclusion, median (IQR) & $67(58-73)$ & $50(43-60)$ \\
\hline Sex, male, n (\%) & $186(56.0)$ & $14(53.8)$ \\
\hline Comorbidity, $\mathrm{n}(\%)^{*}$ & $251(76.3)$ & $25(96.2)$ \\
\hline Vascular & $111(33.7)$ & $7(26.9)$ \\
\hline Malignancy & $67(20.4)$ & $6(23.1)$ \\
\hline Cardiac & $66(20.1)$ & $6(23.1)$ \\
\hline Diabetes mellitus & $61(18.5)$ & $5(19.2)$ \\
\hline Gastrointestinal & $49(14.9)$ & $6(23.1)$ \\
\hline Pulmonary & $39(11.9)$ & $3(11.5)$ \\
\hline Neurologic/psychiatric & $29(8.8)$ & $14(53.8)$ \\
\hline Endocrine & $19(5.8)$ & $2(7.7)$ \\
\hline Other & $78(23.7)$ & $11(42.3)$ \\
\hline Etiology of CP, n (\%) & $\mathrm{N} / \mathrm{A}$ & \\
\hline Alcoholic & & $15(57.7)$ \\
\hline Idiopathic & & $8(30.8)$ \\
\hline Hereditary & & $2(7.7)$ \\
\hline Metabolic & & $1(3.8)$ \\
\hline ECOG score, $\mathrm{n}(\%)^{*}$ & & N/A \\
\hline ECOG 0 & $166(52.5)$ & \\
\hline ECOG 1 & $116(36.7)$ & \\
\hline ECOG 2 & $29(9.2)$ & \\
\hline ECOG 3 & $5(1.6)$ & \\
\hline $\begin{array}{l}\text { Intoxications at baseline } \\
\text { in } \mathrm{CP}, \mathrm{n}(\%)\end{array}$ & $\mathrm{N} / \mathrm{A}$ & \\
\hline Smoking, yes & & $22(84.6)$ \\
\hline Alcohol consumption, yes & & $2(7.7)$ \\
\hline \multicolumn{3}{|c|}{ Indication for surgery, $\mathrm{n}(\%)(>1$ possible $)$} \\
\hline Suspected (pre)malignant disease & $332(100)$ & 0 \\
\hline Pain & 0 & $22(84.6)$ \\
\hline Complications & 0 & $6(23.1)$ \\
\hline Other & 0 & $4(15.4)$ \\
\hline Resection, n (\%) & & $\mathrm{N} / \mathrm{A}$ \\
\hline Yes & $275(82.8)$ & \\
\hline No & $57(17.2)$ & \\
\hline \multicolumn{3}{|l|}{ Type of surgery $\left(\mathrm{N}_{\mathrm{tot}}=300^{\dagger}\right), \mathrm{n}(\%)$} \\
\hline PPPD & $123(44.7)$ & 0 \\
\hline Classical Whipple & $86(31.3)$ & $2(8.0)$ \\
\hline Distal pancreatectomy & $55(20.0)$ & $4(16.0)$ \\
\hline Frey procedure & $\mathrm{N} / \mathrm{A}$ & $11(44.0)$ \\
\hline Pancreatojejunostomy & $\mathrm{N} / \mathrm{A}$ & $7(28.0)$ \\
\hline Other & $11(4.0)$ & $1(4.0)$ \\
\hline \multicolumn{3}{|l|}{$\begin{array}{l}\text { Histological diagnosis }\left(\mathrm{N}_{\mathrm{tot}}=300^{\dagger}\right) \text {, } \\
\mathrm{n}(\%)^{*}\end{array}$} \\
\hline Pancreatic ductal adenocarcinoma & 119 (43.9) & 0 \\
\hline Pancreatic NET & $27(10.0)$ & 0 \\
\hline Cholangiocarcinoma & $26(9.6)$ & 0 \\
\hline Carcinoma of papilla of Vater & $25(9.2)$ & 0 \\
\hline IPMN & $22(8.1)$ & 0 \\
\hline Duodenal carcinoma & $8(3.0)$ & 0 \\
\hline Chronic pancreatitis & $6(2.2)$ & $23(92.0)$ \\
\hline Other & $43(15.9)$ & $2(8.0)$ \\
\hline
\end{tabular}

TABLE 2. (Continued)

\begin{tabular}{|c|c|c|}
\hline Characteristics & $\begin{array}{l}\text { (Pre-)Malignant } \\
\text { Disease }(n=332)\end{array}$ & $\begin{array}{c}C P \\
(n=26)\end{array}$ \\
\hline $\begin{array}{l}\text { Positive lymph nodes in patients } \\
\text { undergoing resection, } \mathrm{n}(\%)\end{array}$ & & N/A \\
\hline $\begin{array}{l}\text { Pancreatic ductal adenocarcinoma } \\
\left(\mathrm{N}_{\text {tot }}=119\right)\end{array}$ & $85(71.4)$ & \\
\hline Cholangiocarcinoma $\left(\mathrm{N}_{\text {tot }}=26\right)$ & $17(65.4)$ & \\
\hline $\begin{array}{l}\text { Carcinoma of papilla of Vater } \\
\left(\mathrm{N}_{\text {tot }}=25\right)\end{array}$ & $14(56.0)$ & \\
\hline Duodenal carcinoma $\left(\mathrm{N}_{\mathrm{tot}}=8\right)$ & $6(75.0)$ & \\
\hline Distant metastases, $\mathrm{n}(\%)^{*}$ & & N/A \\
\hline Yes & $36(11.3)$ & \\
\hline No & $284(88.8)$ & \\
\hline Neoadjuvant treatment, $\mathrm{n}(\%)$ & & N/A \\
\hline No & $301(90.6)$ & \\
\hline Chemotherapy & $12(3.6)$ & \\
\hline Chemoradiotherapy & $18(5.4)$ & \\
\hline Radiotherapy & $1(0.3)$ & \\
\hline $\begin{array}{l}\text { Adjuvant treatment recommended, } \\
\text { in patients undergoing resection, } \mathrm{n}(\%)\end{array}$ & & N/A \\
\hline $\begin{array}{l}\text { Pancreatic ductal adenocarcinoma } \\
\left(\mathrm{N}_{\text {tot }}=119\right)^{*}\end{array}$ & $97(82.2)$ & \\
\hline Cholangiocarcinoma $\left(\mathrm{N}_{\text {tot }}=26\right)$ & $1(3.8)$ & \\
\hline $\begin{array}{l}\text { Carcinoma of papilla of Vater } \\
\left(\mathrm{N}_{\text {tot }}=25\right)\end{array}$ & $1(4.0)$ & \\
\hline Duodenal carcinoma $\left(\mathrm{N}_{\mathrm{tot}}=8\right)$ & $2(25.0)$ & \\
\hline
\end{tabular}

*Unknown in less than $5 \%$ of the patients.

${ }^{\dagger}$ A total of 275 patients with (pre-)malignant disease and 25 patients with $\mathrm{CP}$ (in 1 patient with $\mathrm{CP}$, the exploratory laparotomy was aborted, as there were no signs of $\mathrm{CP}$ during surgery).

ECOG indicates Eastern Cooperative Oncology Group; IPMN, intraductal papillary mucinous neoplasm; IQR, interquartile range; N/A, not applicable; PPPD, pylorus preserving pancreatoduodenectomy.

optimal workflows. A second challenge is gaining and maintaining the trust and commitment of all participating centers. Fortunately, the Netherlands has a strong infrastructure for multicenter collaboration, in part due to the establishment of the DPCG and DPSG. However, each center puts a lot of effort in the collection of the biomaterials and data and wants to ensure that samples are used for the most relevant projects. To at least partially overcome this challenge, a nationwide multidisciplinary scientific board was established within the working groups to assess each research proposal in a plenary meeting. A third difficulty is to combine ongoing institutional research projects and local biobanks with the Dutch Pancreas Biobank. Multidisciplinary collaboration and discussing the distribution of tumor tissue samples over different projects were helpful. The last challenge is costs associated with the project, not only for the materials for sample collection and storage but also for manpower to manage the processes. Because of the grant provided by the Dutch Cancer Society, we are able to cover part of the costs, but the remainder needs to be covered by the participating hospitals. A future aim is to obtain structural (governmental) funding.

Strengths of the Dutch Pancreas Biobank include the rigorous sampling protocol and the systematic collection of follow-up blood. ${ }^{2}$ In addition, the harmonized biomaterial collection across centers is rather unique. This is possible because of the infrastructure and expertise of the PSI. ${ }^{23}$ Moreover, the strong intention of multidisciplinary and multicenter collaboration in the Netherlands 
will ensure long-term fruitful cooperation between the participating centers. Moreover, because of this collaboration, not only the university centers participate in the project but also several nonacademic hospitals. This not only makes it possible to collect a larger amount of samples but also ensures that the subset of patients included in the biobank is representative of the total population. Last, not only patients with pancreatic cancer but also patients with periampullary tumors, NETs, and CP are included, which is not the case in many other biobanks.

A limitation of this project is that we will be unable to collect follow-up samples of the subset of patients who return to their referring hospital for follow-up after surgery. In addition, only patients who planned to undergo resection and hence with earlier stage of disease are included. Therefore, we plan to expand the biobank to patients with locally advanced and metastatic disease. Blood collection will be feasible in these patients, but obtaining high-quality tumor tissue samples from these patients will be more difficult; tumor biopsies from the primary tumor or metastatic sites usually contain a limited amount of tumor tissue material, which is mostly reserved for diagnostic purposes. However, in the Netherlands, we have already gained experience with the collection and biobanking of tumor material during endoscopic procedures. ${ }^{36}$ A known issue in pancreatic cancer tissue samples is the often low percentage of tumor cells in the samples. Microscopically, pancreatic cancer is characterized by an irregular pattern of tumor cells in abundant desmoplastic stroma. ${ }^{37,38}$ This hampers genomic analyses, especially mutation calling, copy number alteration, and methylation analyses. Another problem is intratumor heterogeneity on both the morphological and genomic levels. ${ }^{39}$ Sampling from multiple regions of the tumor may be a strategy to at least partially overcome this problem, but this is not always feasible because of tumor size and location.

A near future goal of the Dutch Pancreas Biobank is to collect samples in all 13 participating centers, which will lead to a fast growth of the number of samples. In the Netherlands, around 1000 patients per year undergo pancreatic surgery (all types of procedures and indications), and $75 \%$ is performed in the 13 centers participating in the biobank. As the great majority of the patients agree to participate in the project, it will be possible to collect samples of hundreds of patients per year. The desired next step would be to include patients with locally advanced and metastatic disease to make it possible to perform future studies with materials of patients with more advanced stages of the disease. The ultimate goal is to use this experience to embed high-quality biobanking into daily routine clinical practice to facilitate "real-time" molecular profiling to achieve personalized care for patients with pancreatic and periampullary diseases. Examples of such projects are the Precision-Panc initiative in the UK and the Precision Promise initiative in the United States.

In conclusion, large-scale biobanks are essential in an era focused on development of personalized treatment based on biomarkers and genomic signatures. Going forward, we would like the Dutch Pancreas Biobank within the PSI to support high-quality research projects, both nationally and internationally.

\section{ACKNOWLEDGMENT}

This biobank is part of the Parelsnoer Institute, an initiative of the Dutch Federation of University Medical Centers (http:// www.parelsnoer.org).

\section{REFERENCES}

1. Siegel RL, Miller KD, Jemal A. Cancer statistics, 2015. CA Cancer J Clin. 2015;65:5-29.

2. Philip PA, Mooney M, Jaffe D, et al. Consensus report of the national cancer institute clinical trials planning meeting on pancreas cancer treatment. J Clin Oncol. 2009;27:5660-5669.
3. Neoptolemos JP, Palmer DH, Ghaneh P, et al. Comparison of adjuvant gemcitabine and capecitabine with gemcitabine monotherapy in patients with resected pancreatic cancer (ESPAC-4): a multicentre, open-label, randomised, phase 3 trial. Lancet. 2017;389:1011-1024

4. Vincent A, Herman J, Schulick R, et al. Pancreatic cancer. Lancet. 2011; 378:607-620.

5. Conroy T, Desseigne F, Ychou M, et al. FOLFIRINOX versus Gemcitabine for Metastatic Pancreatic Cancer. N Engl J Med. 2011;364:1817-1825.

6. Von Hoff DD, Ervin T, Arena FP, et al. Increased survival in pancreatic cancer with nab-paclitaxel plus gemcitabine. N Engl J Med. 2013;369: 1691-1703.

7. Blanke CD, Demetri GD, von Mehren M, et al. Long-term results from a randomized phase II trial of standard- versus higher-dose imatinib mesylate for patients with unresectable or metastatic gastrointestinal stromal tumors expressing KIT. J Clin Oncol. 2008;26:620-625.

8. Bang YJ, Van Cutsem E, Feyereislova A, et al. Trastuzumab in combination with chemotherapy versus chemotherapy alone for treatment of HER2-positive advanced gastric or gastro-oesophageal junction cancer (ToGA): a phase 3, open-label, randomised controlled trial. Lancet. 2010; 376:687-697.

9. Gianni L, Pienkowski T, Im YH, et al. 5-year analysis of neoadjuvant pertuzumab and trastuzumab in patients with locally advanced, inflammatory, or early-stage HER2-positive breast cancer (NeoSphere): a multicentre, open-label, phase 2 randomised trial. Lancet Oncol. 2016;17:791-800.

10. Knudsen ES, O'Reilly EM, Brody JR, et al. Genetic diversity of pancreatic ductal adenocarcinoma and opportunities for precision medicine. Gastroenterology. 2016;150:48-63.

11. Moore MJ, Goldstein D, Hamm J, et al. Erlotinib plus gemcitabine compared with gemcitabine alone in patients with advanced pancreatic cancer: a phase III trial of the National Cancer Institute of Canada Clinical Trials Group. J Clin Oncol. 2007;25:1960-1966.

12. O'Hayer KM, Brody JR. Personalized therapy for pancreatic cancer: Do we need better targets, arrows, or both? Discov Med. 2016;21:117-123.

13. Chandrasegaram MD, Chen JW, Price TJ, et al. Advances in molecular pathology and treatment of periampullary cancers. Pancreas. 2016; 45:32-39.

14. Primrose JN, Fox R, Palmer DH, et al. Adjuvant capecitabine for biliary tract cancer: The BILCAP randomized study. 2017 ASCO Annual Meeting. J Clin Oncol. 2017;35(suppl 15):abstract 4006.

15. Erdmann JI, Eskens FA, Vollmer CM, et al. Histological and molecular subclassification of pancreatic and nonpancreatic periampullary cancers: implications for (neo) adjuvant systemic treatment. Ann Surg Oncol. 2015; 22:2401-2407

16. Oettle H, Neuhaus P, Hochhaus A, et al. Adjuvant chemotherapy with gemcitabine and long-term outcomes among patients with resected pancreatic cancer: the CONKO-001 randomized trial. JAMA. 2013;310: 1473-1481.

17. Majumder S, Chari ST. Chronic pancreatitis. Lancet. 2016;387:1957-1966

18. Hartmann D, Friess H. Surgical approaches to chronic pancreatitis. Gastroenterol Res Pract. 2015;2015:503109.

19. Ravi Kanth V, Nageshwar Reddy D. Genetics of acute and chronic pancreatitis: an update. World J Gastrointest Pathophysiol. 2014;5: $427-437$.

20. Muniraj T, Aslanian HR, Farrell J, et al. Chronic pancreatitis, a comprehensive review and update. Part I: epidemiology, etiology, risk factors, genetics, pathophysiology, and clinical features. Dis Mon. 2014;60:530-550.

21. Sjoquist KM, Chin VT, Chantrill LA, et al. Personalising pancreas cancer treatment: when tissue is the issue. World J Gastroenterol. 2014;20: 7849-7863. 
22. Balarajah V, Ambily A, Dayem Ullah AZ, et al. Pancreatic cancer tissue banks: where are we heading? Future Oncol. 2016;12:2661-2663.

23. Manniën J, Ledderhof T, Verspaget HW, et al. The Parelsnoer Institute: a national network of standardized clinical biobanks in the Netherlands. Open J Bioresour. 2017;4.

24. Navis GJ, Blankestijn PJ, Deegens J, et al. The Biobank of Nephrological Diseases in the Netherlands cohort: the String of Pearls Initiative collaboration on chronic kidney disease in the university medical centers in the Netherlands. Nephrol Dial Transplant. 2014;29: 1145-1150.

25. Haverkamp L, Parry K, van Berge Henegouwen MI, et al. Esophageal and Gastric Cancer Pearl: a nationwide clinical biobanking project in the Netherlands. Dis Esophagus. 2016;29:435-441.

26. Nederkoorn PJ, van Dijk EJ, Koudstaal PJ, et al. The Dutch String-of-Pearls Stroke Study: protocol of a large prospective multicenter genetic cohort study. Int J Stroke. 2015;10:120-122.

27. Parelsnoer Instituut. Standard Operating Procedures. 2016. Available at: http://parelsnoer.org/page/nl/Standaardprocedures. Accessed May 14, 2017.

28. Campa D, Rizzato C, Capurso G, et al. Genetic susceptibility to pancreatic cancer and its functional characterisation: the PANcreatic Disease ReseArch (PANDoRA) consortium. Dig Liver Dis. 2013;45:95-99.

29. Australian Pancreatic Cancer Genome Initiative (APGI). APGI BioResource. 2016. Available at: http://www.pancreaticcancer.net.au. Accessed June 5, 2017.
30. Scarpa A, Chang DK, Nones K, et al. Whole-genome landscape of pancreatic neuroendocrine tumours. Nature. 2017;543:65-71.

31. Bailey P, Chang DK, Nones K, et al. Genomic analyses identify molecular subtypes of pancreatic cancer. Nature. 2016;531:47-52.

32. Waddell N, Pajic M, Patch AM, et al. Whole genomes redefine the mutational landscape of pancreatic cancer. Nature. 2015;518:495-501.

33. Biankin AV, Waddell N, Kassahn KS, et al. Pancreatic cancer genomes reveal aberrations in axon guidance pathway genes. Nature. 2012; 491:399-405

34. Johns Hopkins University. Johns Hopkins Tissue Resource. 2009. Available at: http://pathology.jhu.edu/pc/professionals/shared_tissue_resource. php. Accessed June 5, 2017.

35. University of Liverpool. Liverpool GCLP Facility: Good Clinical Laboratory Practice. Available at: https:/www.liverpool.ac.uk/ translational-medicine/departmentsandgroups/molecular-and-clinicalcancer-medicine/gclp/. Accessed June 5, 2017.

36. Damhofer H, Ebbing EA, Steins A, et al. Establishment of patient-derived xenograft models and cell lines for malignancies of the upper gastrointestinal tract. J Transl Med. 2015;13:115.

37. Hackeng WM, Hruban RH, Offerhaus GJ, et al. Surgical and molecular pathology of pancreatic neoplasms. Diagn Pathol. 2016;11:47.

38. Luchini C, Capelli P, Scarpa A. Pancreatic ductal adenocarcinoma and its variants. Surg Pathol Clin. 2016;9:547-560.

39. Verbeke C. Morphological heterogeneity in ductal adenocarcinoma of the pancreas - Does it matter? Pancreatology. 2016;16:295-301. 